\title{
Performance Evaluation of Two-Equation Turbulence Models for 3D Wing-Body Configuration
}

\author{
Einkeun Kwak*, Namhun Lee* and Seungsoo Lee ${ }^{* *}$ \\ Department of Aerospace Engineering, INHA University, Incheon 402-751, Korea
}

\section{Sang-il Park***}

LG Electronics Inc., Seoul 153-802, Korea

\begin{abstract}
Numerical simulations of 3D aircraft configurations are performed in order to understand the effects of turbulence models on the prediction of aircraft's aerodynamic characteristics. An in-house CFD code that solves 3D RANS equations and two-equation turbulence model equations are used. The code applies Roe's approximated Riemann solver and an AF-ADI scheme. Van Leer's MUSCL extrapolation with van Albada's limiter is also adopted. Various versions of Menter's $k$ - $\omega$ SST turbulence models as well as Coakley's $q-\omega$ model are incorporated into the CFD code. Menter's $k$ - $\omega$ SST models include the standard model, the 2003 model, the model incorporating the vorticity source term, and the model containing controlled decay. Turbulent flows over a wing are simulated in order to validate the turbulence models contained in the CFD code. The results from these simulations are then compared with computational results from the $3^{\text {rd }}$ AIAA CFD Drag Prediction Workshop. Numerical simulations of the DLR-F6 wing-body and wing-body-nacelle-pylon configurations are conducted and compared with computational results of the $2^{\text {nd }}$ AIAA CFD Drag Prediction Workshop. Aerodynamic characteristics as well as flow features are scrutinized with respect to the turbulence models. The results obtained from each simulation incorporating Menter's $k$ - $\omega$ SST turbulence model variations are compared with one another.
\end{abstract}

Key words: Computational fluid dynamics, Turbulence models, Aerodynamic characteristics of an aircraft

\section{Nomenclature}

$b$
$C_{L}$
$C_{D}, C_{D f}, C_{D p}$
$C_{\omega_{1}}, C_{\omega_{2}}, C_{\omega_{3}}, C_{q_{1}}$
$k$
$p$
$q$
$y_{w}$
$\tilde{y}$
$\alpha, \beta, \beta^{*}$
$\mu_{m}$
$\mu_{t}$

Wing Span

Lift coefficient

Total drag, skin friction drag, pressure drag

Model constants of $q$ - $\omega$ turbulence model

Turbulent kinetic energy

Pressure

Turbulent velocity scale

Nearest distance to the wall

Span-wise distance measured from fuselage centerline

Model constants of $k$ - $\omega$ SST turbulence model

Molecular viscosity

Turbulent viscosity
This is an Open Access article distributed under the terms of the Creative Commons Attribution Non-Commercial License (http://creativecommons.org/licenses/by$\mathrm{nc} / 3.0 /$ ) which permits unrestricted non-commercial use, distribution, and reproduction in any medium, provided the original work is properly cited. (c) * Ph. D Student

** Professor, Corresponding author: slee@inha.ac.kr *** Research engineer 


$\begin{array}{ll}\sigma_{S_{1}}, \sigma_{S_{2}} & \text { Model constants of the turbulence models } \\ \omega & \text { Turbulent dissipation rate } \\ \Omega & \text { Absolute value of vorticity }\end{array}$

\section{Introduction}

Computational Fluid Dynamics (CFD) is a major research tool for aircraft design and analysis. However, predicting aerodynamic characteristics is difficult because of aircraft's complex flow features and configurations. Turbulent flow comprises the majority of the flows around an aircraft; it is often accompanied by large separations, wall shear layers, and shocks. Resolving the entire range of turbulent length scales is necessary for accurately analyzing the flow. However, the high computational expenses connected to Direct Numerical Simulation (DNS) forces us to use some sort of turbulence modeling in the simulation of complex flows around aircraft. The Reynolds Averaged Navier-Stokes (RANS) equations are more effective than Large Eddy Simulation (LES) for turbulence modeling. However, the choice of turbulence models strongly influences the RANS solutions. Thus, selecting an appropriate turbulence model requires special care and attention.

The aerodynamic characteristics of an aircraft were researched at the AIAA CFD Drag Prediction Workshops (DPW). At the $2^{\text {nd }}$ AIAA CFD DPW (DPW-2) [1], the DLR-F6 wing-body (WB) model and wing-body-nacellepylon (WBNP) model were selected for drag prediction. Experimental data and results from the participants in DPW2 are presented on the website [1] as well as at the $42^{\text {nd }}$ AIAA Reno conference. At the $3^{\text {rd }}$ AIAA CFD DPW (DPW-3) [2], the DLR-F6 wing-body with FX2B fairing transport model and wing-only models (DPW-W1 and W2) were selected to predict aircraft forces and moments. The FX2B fairing reduced separation at the wing-body juncture. DPW-W1 and $\mathrm{W} 2$ are transonic wing models suitable for validating the code. Experimental data and numerical results are summarized on the website [2].

The purpose of this paper is to evaluate the aerodynamic characteristics of 3D aircraft configurations according to the various turbulence models. This is undertaken using an inhouse CFD code, which is validated through comparison between the numerical results and other computational results for the DPW-W1 model. Numerical simulations of the DLR-F6 WB model and the WBNP model are then performed using various turbulence models including the $q-\omega$ model, the $k$ - $\omega$ SST model, and the variations of the $k-\omega$ SST model. The aerodynamic characteristics and flow features resulting using these models are compared to experimental data and other numerical results.

In this paper, the governing equations and numerical schemes for the mean flow are first presented. Next, various turbulence models are briefly discussed. The simulation results of the DPW-W1 model are presented to show the validity of the code. The aerodynamic characteristics of the DLR-F6 WB and WBNP models are presented and compared with other research results.

\section{Numerical methods}

\subsection{Governing Equations and Numerical schemes}

Three-dimensional RANS equations and the two-equation turbulence model equations are chosen as the governing equations for the simulation of the turbulent flow around an aircraft. The equations are

$$
\frac{\partial W}{\partial t}+\frac{\partial E}{\partial x}+\frac{\partial F}{\partial y}+\frac{\partial G}{\partial z}=\frac{\partial E_{v}}{\partial x}+\frac{\partial F_{v}}{\partial y}+\frac{\partial G_{v}}{\partial z}+S
$$

where $W$ denotes the conservative flow variable vector, $E, F$ and $G$ are the inviscid flux vectors; $E_{v}, F_{v}$ and $G_{v}$ are the viscous flux vectors in each spatial direction. $S$ is the source term vector from the turbulence model equations. The conservative flow variable vector and flux vectors are defined as

$$
\begin{aligned}
& W=\left[\begin{array}{lllllll}
\rho & \rho u & \rho v & \rho w & e & \rho s_{1} & \rho s_{2}
\end{array}\right]^{T}, \\
& E=\left[\begin{array}{lllllll}
\rho u & \rho u^{2}+p & \rho u v & \rho u w & (e+p) u & \rho u s_{1} & \rho u s_{2}
\end{array}\right]^{T},(3) \\
& F=\left[\begin{array}{lllllll}
\rho v & \rho v u & \rho v^{2}+p & \rho v w & (e+p) v & \rho v s_{1} & \rho v s_{2}
\end{array}\right]^{T},(4) \\
& G=\left[\begin{array}{lllllll}
\rho w & \rho w u & \rho w v & \rho w^{2}+p & (e+p) w & \rho w s_{1} & \rho w s_{2}
\end{array}\right]^{T} \\
& E_{v}=\left[\begin{array}{llllll}
0 & \tau_{x x} & \tau_{x y} & \tau_{x z} & \Omega_{x} & \left(\mu_{m}+\sigma_{s_{1}} \mu_{t}\right) \frac{\partial s_{1}}{\partial x}
\end{array} \quad\left(\mu_{m}+\sigma_{s_{2}} \mu_{t}\right) \frac{\partial s_{2}}{\partial x}\right]^{T},(6)
\end{aligned}
$$

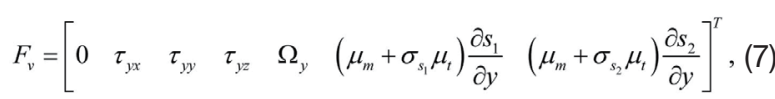

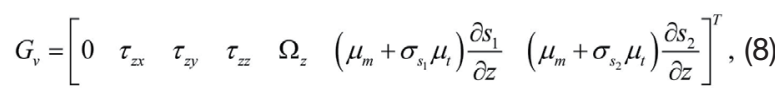


where $\rho, u, v, w, e, s_{1}$ and $s_{2}$ are the density, velocity components in each directions, specific total energy, and turbulence variables, respectively. $\tau_{i j}$ and $\Omega_{i}$ are the total stress tensor and the total energy flux vector, respectively. The equation of state for an ideal gas is used.

The governing equations are solved using a cell centered Finite Volume Method (FVM). Roe's approximated Riemann solver [3] is used for computing the inviscid flux; the central difference method is employed for the viscous flux. Van Leer's MUSCL extrapolation [4] with a limiter is used to obtain second-order accuracy while maintaining the Total Variation Diminishing (TVD) property. An Approximate Factorization-Alternative Direction Implicit (AF-ADI) scheme [5] is used for the steady-state solution.

\subsection{Turbulence models}

Coakley's $q-\omega$ model [6] and the various forms of Menter's $k-\omega$ SST model [7] are used to compute the turbulence quantities.

\subsubsection{Coakley's $q$ - $\omega$ model}

The turbulent velocity scale, $q$ and the specific dissipation rate, $\omega$ are used as the turbulence variables for Coakley's $q$ - $\omega$ model in order to estimate the eddy viscosity. The model used in this study is the baseline model with an added compressibility correction. The eddy viscosity is computed through the Prandtl-Kolmogorov relation

$$
\mu_{t}=C_{\mu} D_{q} \frac{\rho q^{2}}{\omega},
$$

where $C_{\mu}=0.09$. The damping function is defined by

$$
D_{q}=1-\exp \left(-0.022 R_{q}\right), R_{q}=\frac{\rho q y}{\mu},
$$

where $y$ is the normal distance from the nearest solid wall. The source term vector is written as:

$$
\begin{gathered}
S=\left(\begin{array}{c}
C_{q 1}\left(C_{\mu} \frac{\tilde{S}}{\omega^{2}}-\frac{2}{3} \frac{D}{\omega}-1\right) \rho \omega q \\
{\left[C_{\omega 1}\left(C_{\mu} \frac{\tilde{S}}{\omega^{2}}-C_{\omega 3} \frac{D}{\omega}\right)-C_{\omega 2}\right] \rho \omega}
\end{array}\right), \\
\tilde{S}=\left(u_{i, j}+u_{j, i}\right)-\frac{2}{3} D^{2}, D=\frac{\partial u}{\partial x}+\frac{\partial v}{\partial y}+\frac{\partial w}{\partial z} .
\end{gathered}
$$

\subsubsection{Menter's $k$ - $\omega$ SST Model (SST) and Its Various Versions}

Menter's $k$ - $\omega$ SST Model is developed to predict accurately aeronautical flow exhibiting strong adverse pressure gradients and separation. The eddy viscosity of the SST model is

$$
\begin{aligned}
& v_{t}=\frac{a_{1} k}{\max \left(a_{1} \omega ; \Omega F_{2}\right)}, \\
& F_{2}=\tanh \left(\arg _{2}^{2}\right), \\
& \arg _{2}=\max \left(2 \frac{\sqrt{k}}{0.09 \omega y_{w}} ; \frac{500 v}{y_{w}^{2} \omega}\right) .
\end{aligned}
$$

The source term vector is defined as

$$
\begin{aligned}
& S=\left(\begin{array}{c}
P-\beta^{*} \rho \omega k \\
\frac{\alpha}{v_{t}} P-\beta \rho \omega^{2}+2\left(1-F_{2}\right) \frac{\rho \sigma_{\omega 2}}{\omega} \frac{\partial k}{x_{j}} \frac{\partial \omega}{\partial x_{j}}
\end{array}\right), \\
& P=\tau_{i j} \frac{\partial u_{j}}{\partial x_{j}} .
\end{aligned}
$$

The other model constants are determined with blending function $F_{1}$.

\subsubsection{SST Model with the Vorticity Source Term (SST-V) [8]}

The vorticity magnitude is more easily computed in comparison to the exact source term. The vorticity magnitude is generally very close to the exact source term in simple boundary layer flows. In this model, the term $P$ is redefined as

$$
P=\mu_{t} \Omega^{2}-\frac{2}{3} \rho k \delta_{i j} \frac{\partial u_{i}}{\partial x_{j}} .
$$

\subsubsection{SST Model from 2003 (SST-2003) [9]}

The SST-2003 model redefines the eddy viscosity term. Eddy viscosity is computed using the strain invariant rather than the vorticity magnitude.

$$
v_{t}=\frac{a_{1} k}{\max \left(a_{1} \omega ; \hat{S} F_{2}\right)},
$$

where

$$
\hat{S}=\sqrt{2 S_{i j} S_{i j}}, S_{i j}=\frac{1}{2}\left(\frac{\partial u_{i}}{\partial x_{j}}+\frac{\partial u_{j}}{\partial x_{i}}\right) .
$$

While the production limiter is used for the $k$ equation in standard model, the production limiter is applied to both 
$k$ and $\omega$ equations. Other minor changes can be found in Menter et al.'s study [9].

\subsubsection{SST Model with Controlled Decay (SST-sust)} [10]

For external aeronautical flows, the SST model with controlled decay (SST-sust) prevents the non-physical decay of the turbulence variables in the free-stream. Sustaining terms are added to the source term in the turbulence equations; the changed source term vector is therefore redefined as follows:

$$
S_{s u s t}=S+\left(\begin{array}{c}
\beta^{*} \rho \omega_{a m b} k_{a m b} \\
\beta \rho \omega_{a m b}^{2}
\end{array}\right)
$$

\section{Computational results}

\subsection{DPW-W1 Configuration}

Numerical simulations of the DPW-W1 configuration are conducted to validate the CFD code. The results are then compared with computational results by Tinoco [2]. The Mach number of the flow is 0.76 and the Reynolds number is $5.0 \times 10^{6}$ based on a Mean Aerodynamic Chord (MAC) length of $197.556 \mathrm{~mm}$. The angle of attack (AOA) is 0.5 degree. The grid used in Tinoco's research is employed in these simulations. The $k$ - $\omega$ SST model and the $q-\omega$ model are used for turbulent viscosity. The geometry of the wing and the two span locations $\eta=2 \tilde{y} / b$ used for the comparisons of pressure distributions are presented in Fig. 1. Pressure coefficient distributions around the upper surface are also shown. Comparisons between the pressure distributions

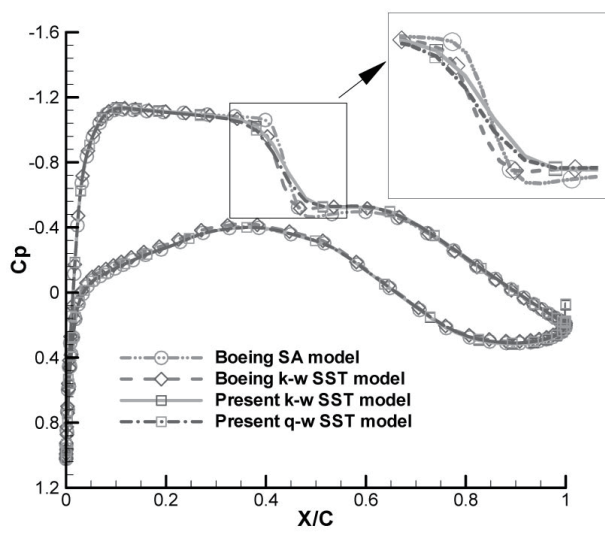

(a) $\eta=0.420$ in the two positions are shown in Fig. 2; the pressure distributions correlate well with Tinoco's results except close to the shock locations, where there are slight differences in pressure. However, determining which results are more accurate is difficult without experimental data.

Drag polars computed by the current code are compared with Tinoco's results in Fig. 3; there is good agreement in the pressure drags, while the calculated skin friction drags differ. Thus, the difference between the total drag coefficients is due to discrepancies in the values of skin friction drag. The pressure differences near the shock locations have little effect on the aerodynamic coefficients. Relative to Tinoco's results, the total drag in the $k$ - $\omega$ SST model is lower, while the total drag in the $q-\omega$ model is higher. The differences in the total drag at $C_{L}=0.5$ are within \pm 10 drag counts. The three drag polars do not show significant differences for positive incidences.

\subsection{DLR-F6 Configurations}

Changes in aerodynamic characteristics with respect to turbulence models are explored through simulations of the

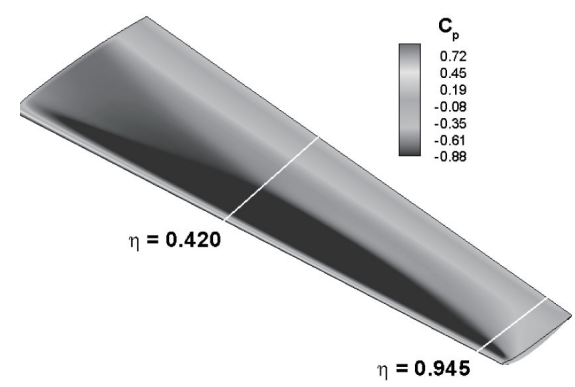

Fig. 1. DPW-W1 geometry and pressure coefficients with the positions of $\eta\left(\mathrm{AOA}: 0.5^{\circ}\right)$

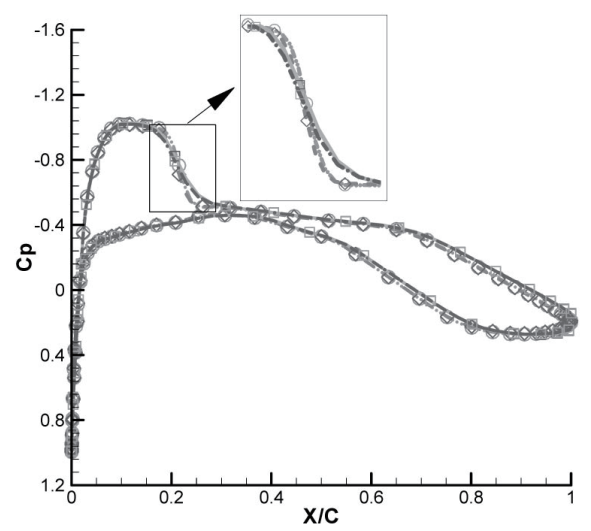

(b) $\eta=0.945$

Fig. 2. Comparison of pressure distributions at two span locations (DPW-W1, AOA : $0.5^{\circ}$ ) 


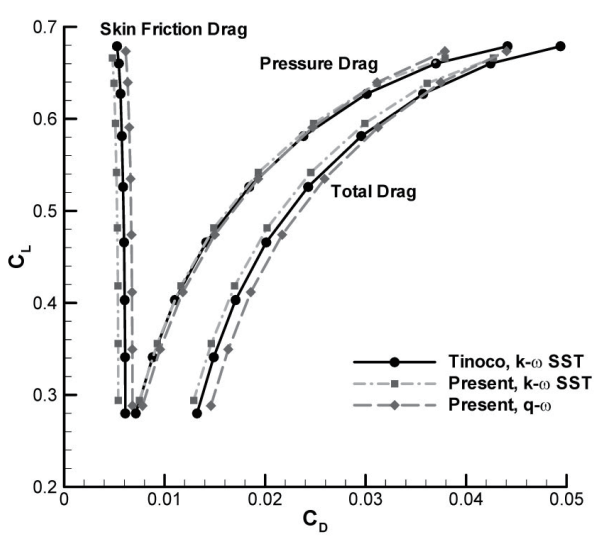

Fig. 3. Comparison of drag polars of DPW-W1

flows around the DLR-F6 WB configuration. The compared turbulence models are the $q$ - $\omega$ model, the $k$ - $\omega$ SST model, and variations of the SST model. The Mach number is 0.75 and the lift coefficient is 0.5 under the design cruise condition. The simulations are performed at a fixed angle of attack, because matching the angle of attack provided more accuracy than matching the lift coefficient in most of the DPW results. The Reynolds number based on the MAC length is $3.0 \times 10^{6}$. The medium (3.9 million grid points) and the fine ( 8.9 grid points) grid systems made by Tinoco [1] are used in the simulations. Magnified views of the grid systems near the wing-body junction are depicted in Fig. 4 . Figure. 5 compares the drag polars obtained with the two grid systems. In the figure, the skin friction drag, the pressure drag, and the total drag are plotted. As can be seen in Fig. 5, the shapes of the drag polars do not change as the grid density increases. The points on the drag polars computed with the fine grid system at an AOA lie on the lines generated with the medium grid system. Since the performance of an aircraft depends only upon the shape of the drag polar, it is decided that the subsequent computations of the DLR-F6 WB are performed with the medium grid system only.

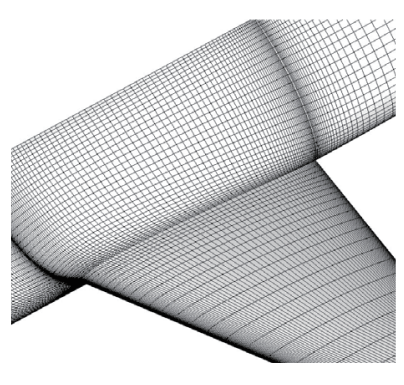

(a) Medium grid

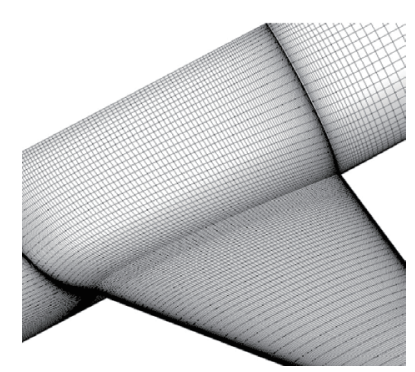

(b) Fine grid
Fig. 4. Surface grids of medium and fine grid systems near the wingbody junction (WB)

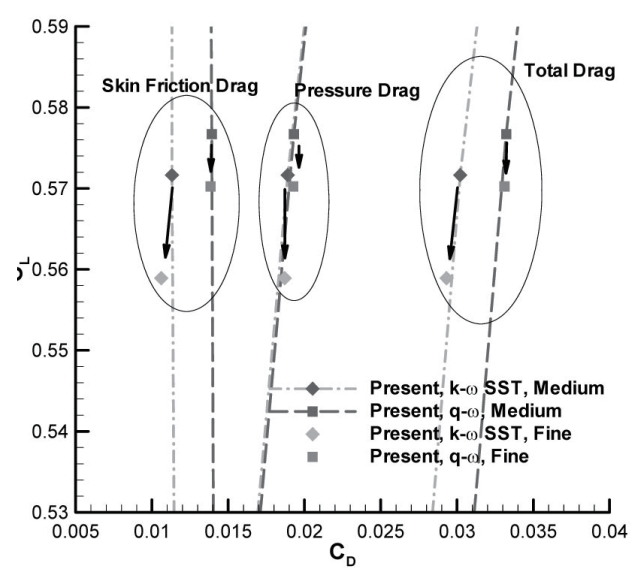

Fig. 5. Change of drag and lift by grid resolutions (WB, AOA : $0.5^{\circ}$ )

The DLR-F6 WB configuration and the pressure coefficient distribution along the surface are presented in Fig. 6. The span locations of the pressure distributions are also displayed. The figure shows that the shock is clearly established on the upper surface of the wing. The angle of attack and the drag coefficients of the present results and experimental work at the design cruise condition are listed in Tab. 1. A centerline and scatter limits are also presented. The median (Med.) and the scatter limits are computed by statistical methods and used for comparison of the code-tocode scatter [11]. The limits (upper limit : Up., lower limit : Lo.) provide a reasonable estimate of the population mean and the standard deviation of the core solutions. The table shows that the present results fall within the limits. The $q-\omega$ model generates a drag coefficient closer to the experimental results than the $k$ - $\omega$ SST model does. The $k$ - $\omega$ SST model tends to underestimate the drag coefficient, as in the DPW-W1 results. To understand the basis of this tendency, further investigation of the behavior of turbulent viscosity and boundary layer transition is needed.

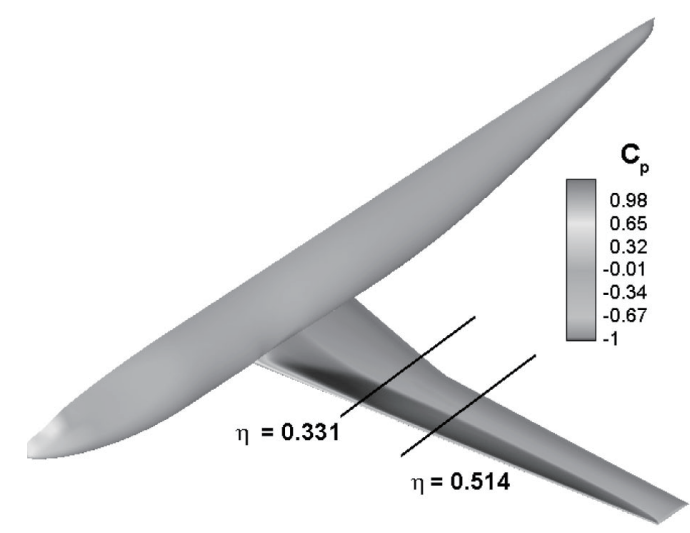

Fig. 6. DLR-F6 WB configuration and pressure coefficients with the positions of $\eta\left(\mathrm{AOA}: 0.5^{\circ}\right)$ 
Table 1. Angles of attack and values of drag at the design cruise condition (AOA : deg, Drag: counts)

\begin{tabular}{|c|c|c|c|c|c|}
\hline Model & $C_{L}$ & $\mathrm{AOA}$ & $C_{D p}$ & $C_{D f}$ & $C_{D}$ \\
\hline Exp. & & 0.52 & - & - & 295 \\
\hline Lo. & & -0.232 & 124 & 86 & 271 \\
\hline Med. & & 0.159 & 165 & 126 & 292 \\
\hline SST & 0.0 & -0.150 & 157 & 115 & 272 \\
\hline$q-\omega$ & & -0.152 & 158 & 141 & 299 \\
\hline Up. & & 0.550 & 206 & 167 & 312 \\
\hline
\end{tabular}

Comparisons of the pressure distributions at the two span positions are presented in the Fig. 7 . At $\eta=0.331$, the pressure distributions of the present results agree well with Tinoco's numerical results and with the experimental data. At $\eta=0.514$, the shock computed by the $k$ - $\omega$ SST model is slightly further downstream than those of other results, whereas the pressure distributions agree well with experiment, except on the upper surface near the shock position. The differences of the shock positions are not significant in comparison with the results obtained at the DPW. The $q$ - $\omega$ model provides a more accurate estimate than the $k$ - $\omega$ SST model, as can be seen in Fig. 7.

In Fig. 8, the drag polars are compared with other numerical results and with experimental data. The drag polars of the pressure drag exhibit little differences between the sets of results, but the skin friction drag shows a larger difference, demonstrating that the turbulence model significantly influences predictions of aerodynamic characteristics. The lowest calculated total drag value is from the $k$ - $\omega$ SST model - 23 drag counts below the experimental result at the design point; the highest is from the $q-\omega$ model - 4 drag counts above the experimental result at the design point. The drag polars computed with the $k$ - $\omega$ SST models, shown in Fig. 9, show little difference; the largest difference of total drag at the design point is 2 drag counts. Therefore, the effect on aerodynamic coefficients of the variations of the $k$ - $\omega$ SST model is insignificant.

Simulations of the WBNP configuration are conducted under the same flow conditions as the WB configuration using Tinoco's medium (6.2 million grid points) and fine (8.7 million grid points) grid systems. Magnified views of the grids on the surface near nacelle and pylon are shown in Fig. 10. The changes of drag and lift using the two grids are shown in Fig. 11. The same movement in the drag polars is found with the fine grid system as in the $\mathrm{WB}$ configuration. The configuration of the WBNP and the pressure distributions around the wing are depicted in Fig. 12. The two $\eta$ locations where the pressure distributions are depicted are also displayed. In Fig. 12, shock becomes clearly established on the upper surface of the wing. In Fig. 13, pressure distributions at the two locations are shown in comparison with the experimental data and Tinoco's computational results. The results with the $q$ - $\omega$ model correlate well with the other results at the two locations. However, the results obtained using the $k$ - $\omega$ SST model show discrepancies in the pressure distribution on the wing's lower surface at $\eta=0.331$ and near the shock position at $\eta=0.514$. In the most simulations, the $k-\omega$ SST model predicts that the shock would be located slightly further downstream from the shock positions from other results. More detailed study is required to identify the cause of the difference.

The stream-traces on the upper surface of the wing near the wing-body junction are shown in Fig. 14. The flow region with the $k$ - $\omega$ SST model is larger than that with the $q-\omega$ model. Similar is observed in the simulations with the fine grid system and the flow separation regions are expanded as grid density increases. Figure 15 compares the streamtraces on the lower surface of the wing near the pylon and the streamlines obtained from the oil flow experiments [1]. The $k-\omega$ SST model predicts an excessive flow separation in comparison with the $q-\omega$ model and the experimental data. Such a tendency has been reported in other investigations [12-16]. Computational results containing AOA matching using the fine grid system show similar discrepancies in the pressure distribution. Similar excessive flow separations around the lower surface of the wing near the pylon are observed with the fine grid, while the solutions obtained using the medium grid correlate well with the experimental data $[12,13]$. The lift matching results also show similar discrepancies in the separation bubble [14-16]. Figure 16 
compares the pressure distributions of previous studies and the present results. According to Klausmeyer's numerical study [17], the separation bubble size on the lower surface of the wing as well as on the wing side-of-body grew as grid density increased. According to May et al. [18], the separation bubble size on the wing side-of-body was influenced by the turbulence model. The separation bubbles predicted by the S-A model and the $k-\omega$ SST model were larger than those predicted by the $k$ - $\omega$ model and the modified $k$ - $\omega$

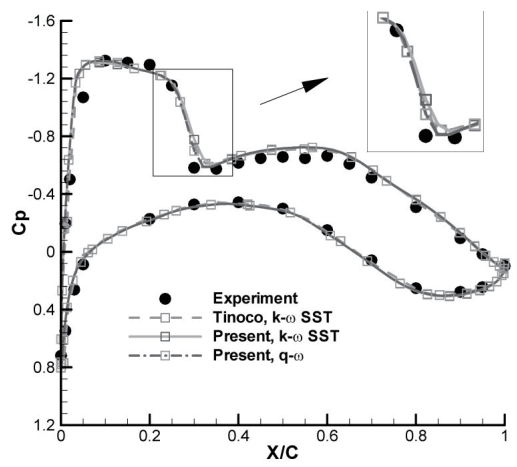

(a) $\eta=0.331$ model. These results suggest that simulation under a certain combination of grid density and turbulence model results in excessive flow separation on the wing surface near the pylon.

The drag polars are compared to Tinoco's results and experimental data in Fig. 17. As in DPW-W1 and WB, significant differences are not observed between the pressure drags, but the skin friction drag coefficients differ from Tinoco's results. Thus, the turbulence model would be the greatest influence on the drag coefficient in the all

Fig. 7. Comparison of pressure distributions at two span locations (WB, $A O A: 0.5^{\circ}$ )

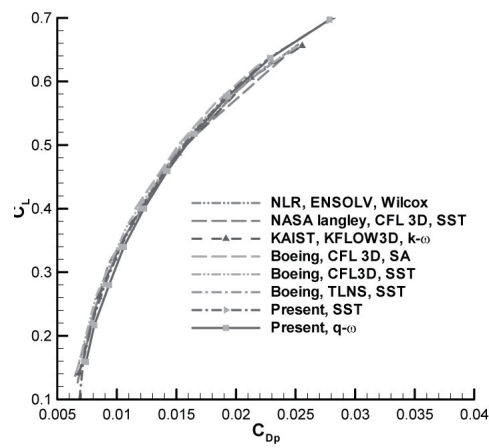

(a) Pressure drag

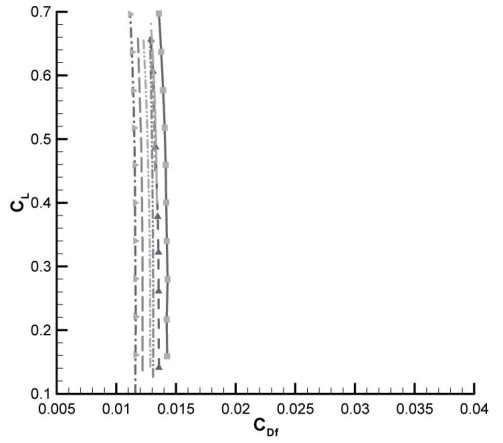

(b) Skin friction drag

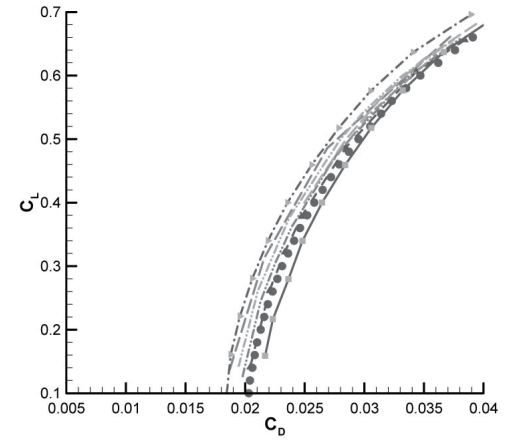

(c) Total drag

Fig. 8. Comparison of the drag polars with other results (WB, Large blue circle : Experiment)

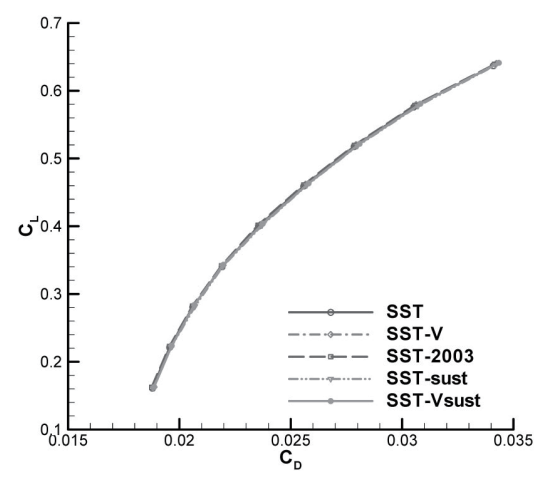

Fig. 9. Comparison of drag polars according to various forms of the SST Model (WB) 
simulations. The skin friction drags are underestimated by the $k$ - $\omega$ SST model in comparison with Tinoco's results, while the skin friction drags are overestimated by the $q-\omega$ model. In comparison with the experimental data, the total drag coefficients obtained from the $k$ - $\omega$ SST model correlate well for negative incidences, but total drag coefficients with the $q$ - $\omega$ model are well matched for positive angles of attack.

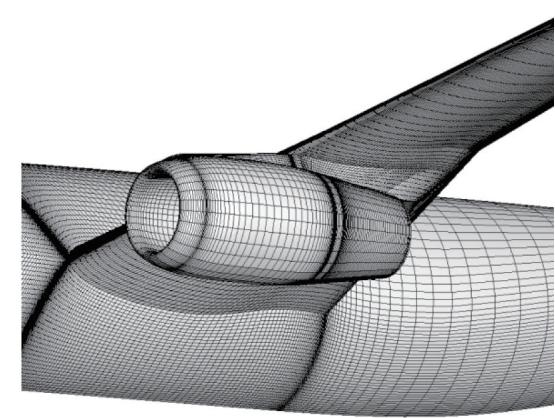

(a) Medium grid

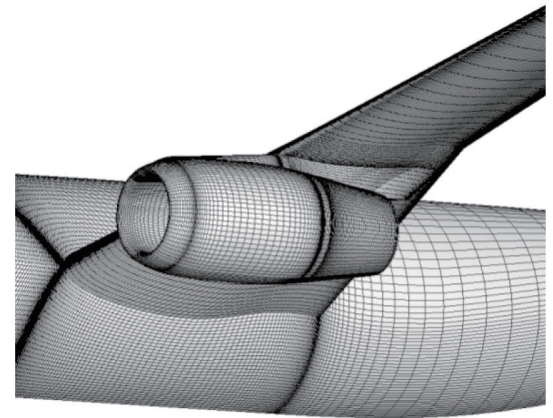

(b) Fine grid

Fig. 10. Surface grids of medium and fine grid systems near the nacelle-pylon (WBNP)

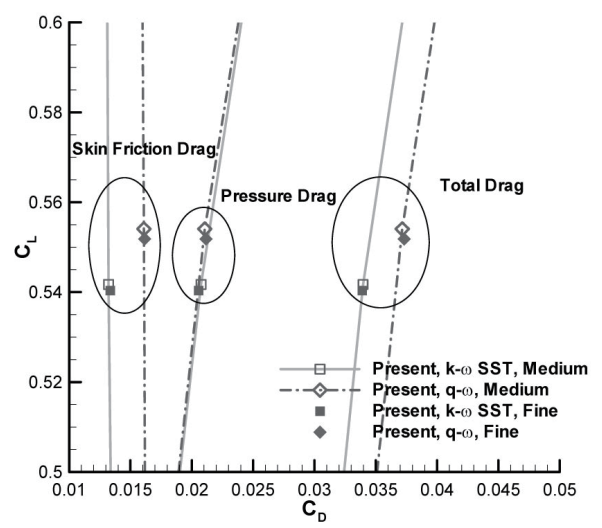

Fig. 11. Changes of drag and lift with grid resolution (WBNP, AOA : 1.0 $0^{\circ}$

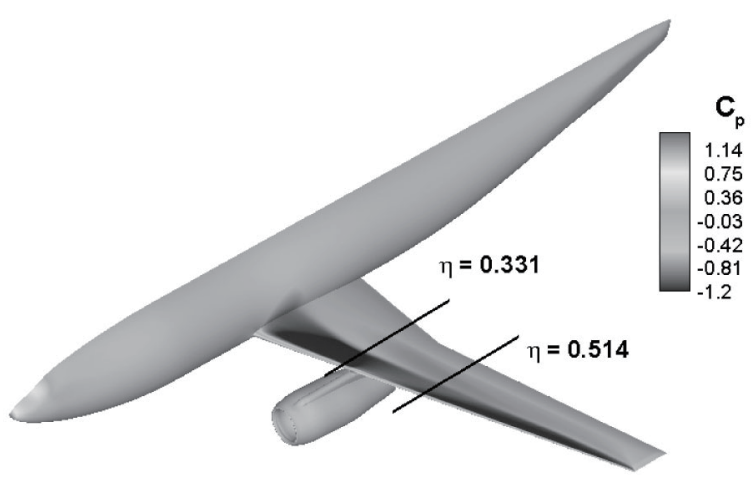

Fig. 12. DLR-F6 WBNP configuration and pressure coefficients distributions (AOA : $\left.1.0^{\circ}\right)$

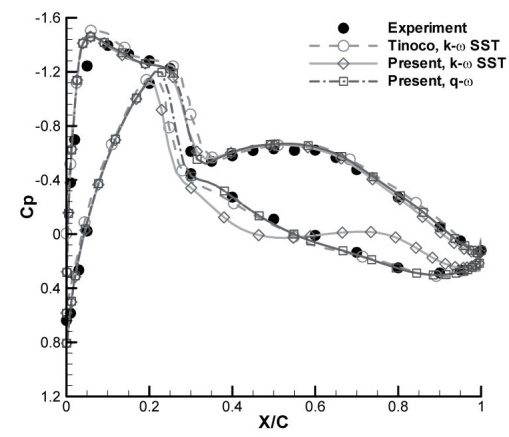

(a) $\eta=0.331$

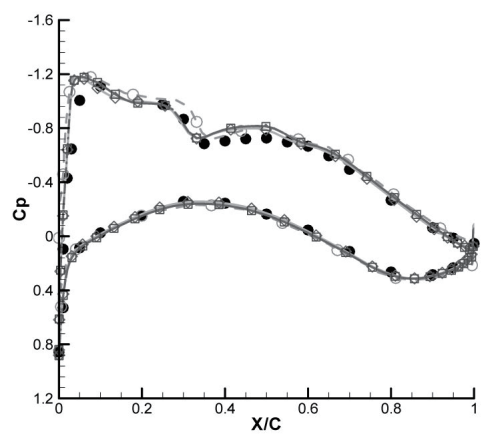

(b) $\eta=0.514$

Fig. 13. Comparison of pressure distributions at two span locations (WBNP, AOA : $1.0^{\circ}$ ) 


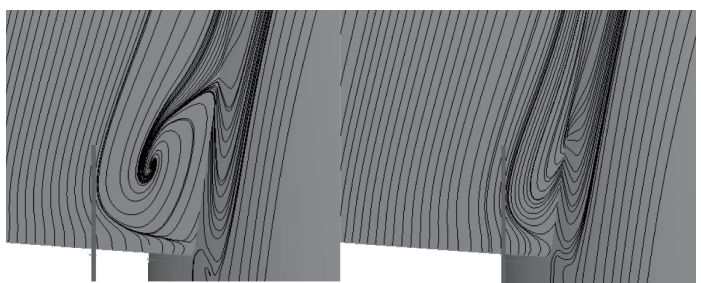

(a) Medium grid (left : $k$ - $\omega$ SST model, right : $q-\omega$ model)

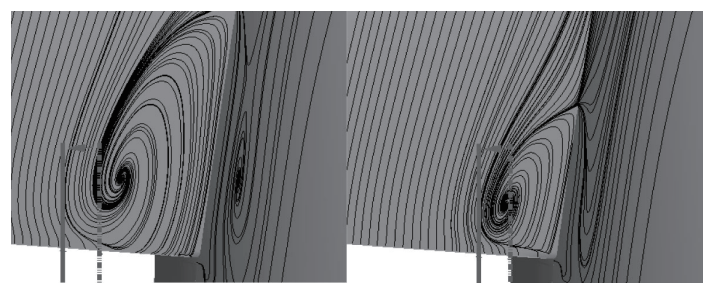

(b) Fine grid (left : $k$ - $\omega$ SST model, right : $q-\omega$ model)

Fig. 14. Comparison of stream-traces near wing-body junction (WBNP, AOA : $1.0^{\circ}$ )

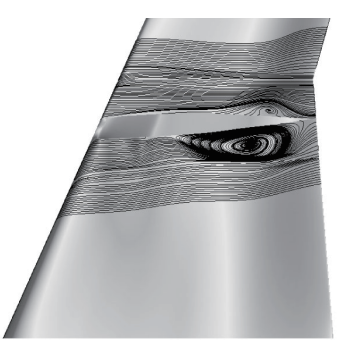

(a) Experiments

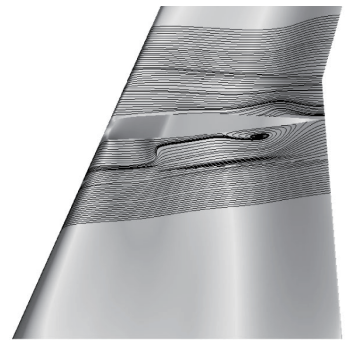

(b) $k$ - $\omega$ SST model

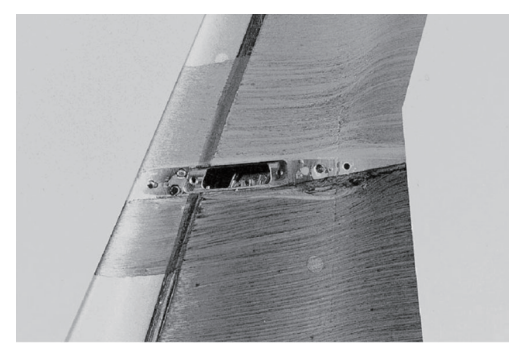

(c) $q$ - $\omega$ model

Fig. 15. Comparison of stream-traces on the wing lower surface near pylon (WBNP, AOA: $1.0^{\circ}$ )

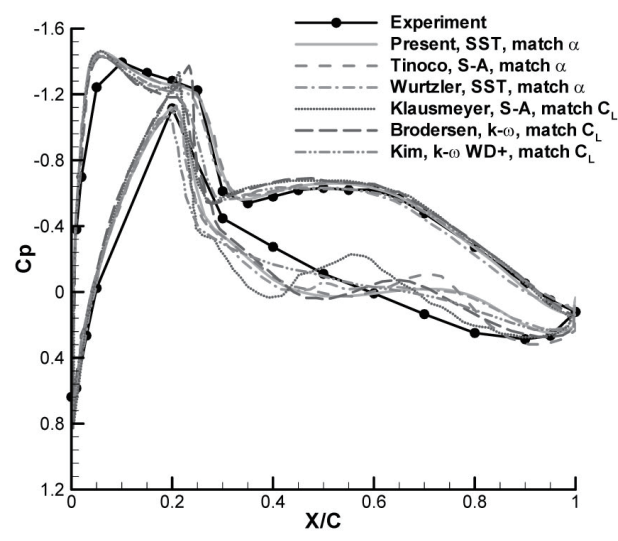

Fig. 16. Comparison of pressure distributions of WBNP $(\eta=0.331)$

\section{Concluding Remarks}

Simulations of aircraft configurations were performed using various turbulence models to understand an aircraft's aerodynamic characteristics. The $q$ - $\omega$ turbulence model, the $k$ - $\omega$ SST turbulence model, and various versions of the SST model were used. The code was verified and validated against previously attained numerical results for flows around the DPW-W1 model. Numerical simulations of the DLR-F6 WB and WBNP configurations were also performed and aerodynamic characteristics were compared with experimental and other computational data. In simulation

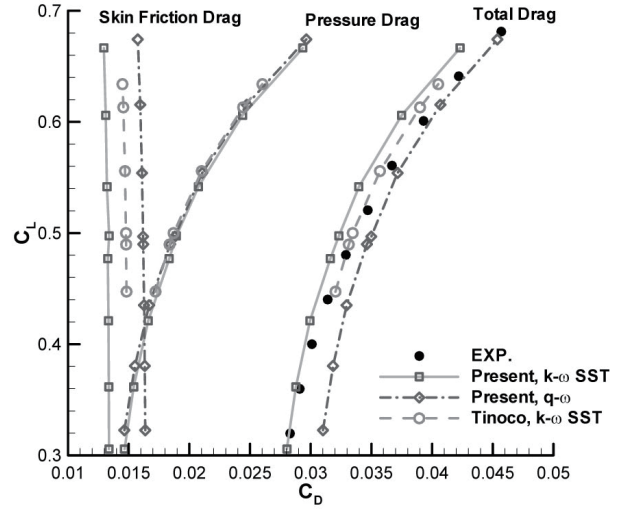

Fig. 17. Comparison of drag polars of WBNP

of the WB configuration, the $k$ - $\omega$ SST model underestimated skin friction drag while the $q$ - $\omega$ model overestimated skin friction drag. Effects on the aerodynamic characteristics induced by variations of the $k$ - $\omega$ SST model proved to be insignificant. In the WBNP configuration simulations, the total drag coefficients computed with the $k$ - $\omega$ SST model correlated well with the experimental data for negative incidences. In contrast, the total drag coefficients obtained from using the $q$ - $\omega$ model were well matched for positive angles of attack. The reason for the excessive flow separation resulting from the use of the $k$ - $\omega$ SST model will be the subject of future work. 


\section{References}

[1] URL: http://aaac.larc.nasa.gov/tsab/cfdlarc/aiaa-dpw/ Workshop2/, Sep. 5, 2011.

[2] URL: http://aaac.larc.nasa.gov/tsab/cfdlarc/aiaa-dpw/ Workshop3/, Sep. 5, 2011.

[3] Roe, R. L., "Approximate Riemann solver, parameter vectors and difference schemes", Journal of Computational Physics, Vol. 43, No. 2, 1981, pp. 357-372.

[4] Van Leer, B., "Towards the ultimate conservative difference scheme. V. A second order sequel to Godunov's method", Journal of Computational Physics, Vol. 32, No. 1, 1976, pp. 101-136.

[5] Beam, R. M., and Warming, R. F., "Implicit numerical methods for the compressible Navier-Stokes and Euler equations", von Karman Institute for Fluid Dynamics Lecture Series, 1982.

[6] Coakley, T. J., "Turbulence modeling Methods for the compressible Navier-Stokes equations", AIAA paper, Paper number 83-1693, 1983.

[7] Menter, F. R., “Two-equation eddy-viscosity turbulence models for engineering applications", AIAA Journal, Vol. 32, No.8, 1994, pp. 1598-1605.

[8] Menter, F. R., "Improved Two-Equation $k$ - $\omega$ Turbulence Models for Aerodynamic Flows", NASA TM 103975, October, 1992.

[9] Menter, F. R., Kuntz, M., and Langtry, R., "Ten Years of Industrial Experience with the SST Turbulence Model", Turbulence, Heat and Mass Transfer 4, edited by K. Hanjalic, Y. Nagano, and M. Tummers, Begell House, Inc., 2003, pp.
625-632.

[10] Spalart, P. R. and Rumsey, C. L., "Effective Inflow Conditions for Turbulence Models in Aerodynamic Calculations", AIAA Journal, Vol. 45, No 10, 2007, pp. 25442553.

[11] Hemsch, M. J. and Morrison, J. H., "Statistical Analysis of CFD Solutions from 2nd Drag Prediction Workshop”, AIAA paper, Paper number 2004-556, 2004.

[12] Tinoco, E. N. and Su, T. B., "Drag Prediction with the ZEUS/CFL3D System”, AIAA paper, Paper number 2004-552, 2004.

[13] Wurtzler, K. E. and Morton, S. A., "Accurate Drag Prediction Using Cobalt", AIAA paper, Paper number 20040395, 2004.

[14] Kim, Y., Park, S. H., Kwon, J. H., and Sung, C., "Drag Prediction of DLR-F6 Using the Turbulent Navier-Stokes Calculations with Multigrid", AIAA paper, Paper number 2004-397, 2004.

[15] Brodersen et al., "Airbus, ONERA, and DLR Results from the 2nd AIAA Drag Prediction Workshop", AIAA paper, Paper number 2004-0391, 2004.

[16] Wurtzler, K. E. and Morton, S. A., "Accurate Drag Prediction Using Cobalt". AIAA paper, Paper number 20040395, 2004.

[17] Klausmeyer, S. M., "Drag, Lift, and Moment Estimates of Transonic Aircraft Using the Navier-Stokes Equations", AIAA paper, Paper number 2004-553, 2004.

[18] May, G., van der Weide, E., Jameson, A., Sriram, and Martinelli, L., "Drag Prediction of the DLR-F6 Configuration", AIAA paper, Paper number 2004-396, 2004. 